\title{
Hydrophilization of PP Resin and ABS Resin by Applying Electrolyzed Sulfuric Acid
}

\author{
Yasushi Umeda ${ }^{1 *}$, Yuki Nakabayashi ${ }^{2}$, Tatsuo Nagai ${ }^{3}$ \\ Katsuhiko Tashiro ${ }^{4}$, Hideo Honma ${ }^{2}$ and Yukihiro Sakamoto ${ }^{5}$ \\ 1 Materials and Surface Engineering Research Institute, Kanto Gakuin University : 1162 -2 Ogikubo, Odawara, Kanagawa \\ 250-0042, Japan \\ 2 Graduate School of Engineering, Kanto Gakuin University : 1162 -2, Ogikubo, Odawara, Kanagawa 250 -0042, Japan \\ 3 MicroAce Co., Ltd. : 1411 -1 Kyode, Hanagashima-cho, Miyazaki 880-0036, Japan \\ 4 Kanto Gakuin University Research advancement and management organization : 1162 -2, Ogikubo, Odawara, Kanagawa \\ 250-0042, Japan \\ 5 Graduate School of Engineering, Chiba Institute of Technology : 2 -17-1 Tsudanuma, Narashino, Chiba 275 -8588, Japan \\ * Corresponding author : umeda@kanto -gakuin.ac.jp
}

\begin{abstract}
Mixture of chromic acid and sulfuric acid has been widely applied for hydrophilization of ABS resin. However, it is difficult to make the hydrophilic surface of PP resin, because chromic acid has poor oxidizing power for PP resin. In addition, due to environmental concerns, alternative technologies that do not use hexavalent chromium are required. The authors have confirmed that PP resin and ABS resin can be hydrophilized with electrolyzed sulfuric acid solution and then be plated to obtain enough adhesion strength in practical use. In this report, focusing on its hydrophilization behavior, we investigated the reason why hydrophilization is created, and assumed that hydrophilization is due to oxygen entering between bonds of carbon and hydrogen to become hydroxyl groups. Key words : PP resin, ABS resin, electrolyzed sulfuric acid, hydrophilization
\end{abstract}

\section{INTRODUCTION}

Plastics have replaced metals as structural materials and parts materials, taking advantages of weight saving, cost reduction, shape freedom, and ease of mass production. In general, it is widely used not only for decoration, but also for exterior and interior parts of automobiles, household electric appliances and so on . At that time, in order to improve rigidity, abrasion resistance, weather resistance, heat resistance, metal plating is often applied to the plastic surface.

Since plastics are nonconductive, it is necessary first to form a metallic film as a conductor on the plastics in order to perform plating. The methods are roughly classified into dry methods and wet methods. There are methods such as chemical vapor deposition (CVD) and physical vapor deposition (PVD) as dry method $\mathrm{s}$, and there are techniques such as electroless nickel plating and electroless copper plating as wet methods. The wet techniques have been adopted so far because the dry methods are mostly to form films under vacuum conditions and are not suitable for mass production and/or application to big parts.

Chromic acid etching is used as a hydrophilization treatment of plastics molded products before metal plating . However, it is difficult to make the hydrophilic surface of polypropylene (hereinafter PP) resin, although possible to hydrophilize acrylonitrile -butadiene-styrene (hereinafter ABS) resin. Because chromic acid has poor oxidizing power for PP resin. In addition, due to environmental concerns, alternative technologies that do not use hexavalent chromium are required [1]. Therefore, we have studied the plating method to ABS resin and PP resin using electrolyzed sulfuric acid (hereinafter ESA) treatment $[2,3]$ for pretreatment of plating process $[4,5]$.

The reason why the hydrophobic surface $\mathrm{s}$ of resin $\mathrm{s}$ can adsorb the catalyst to enable electroless plating is that functional group s are created on the resin surface $s$ and the surfaces become hydrophilic. We report the hydrophilization behavior of $\mathrm{PP}$ resin and ABS resin surface from the viewpoint of functional group $\mathrm{s}$.

\section{EXPERIMENTAL METHODS}

2.1 Sample

(1) PP resin

PP resin plate $\mathrm{S}(50 \mathrm{~mm} \times 50 \mathrm{~mm})$ made by Japan Polypropylene Corp. were used. Since PP resin is excellent in chemical resistance, the amorphous surface becomes crystalline by a swelling treatment [6] with an organic solvent and can be etched by acid easily. For this reason, swelling treatment with xylene ( 80 degree $\quad$-C., 30 minutes) was carried out and heating was carried out at 80 degree-C for 1 hour in the air for drying and stress relaxation before performing ESA treatment. Thereafter, the PP resin plates were immersed for 60 minute $\mathrm{s}, 90$ minutes, or 120 minutes in ESA solution of 120 degree -C whose sulfuric acid concentration was 92 weight percentage and oxidizer concentration is $10 \mathrm{~g} / \mathrm{dm}^{3}$, and they were provided for X-ray Photoelectron Spectrometer (JPS-90SX by JEOL, hereinafter XPS) and Fourier Transform Infrared Spectroscopy (Spectrum One by Perkin Elmer, hereinafter FT-IR) respectively. 
(2) ABS resin

ABS resin plate s $(50 \mathrm{~mm} \times 50 \mathrm{~mm})$ made by Sumitomo Bakelite Co. were immersed for 10 minute s, 20 minute s, and 30 minute $\mathrm{s}$ in ESA solution of 50 degreeC whose sulfuric acid concentration was 75 weight percentage and oxidizer concentration is $10 \mathrm{~g} / \mathrm{dm}^{3}$ respectively, and they were provided for XPS and FT -IR analysis respectively.

\subsection{Analysis}

After the ESA treatment, two kinds of analyzes were performed on all samples; XPS analysis and FT -IR analysis.

\section{RESULTS AND DISCUSSION \\ 3.1 XPS analysis \\ (1) PP resin}

XPS analysis was carried out for all samples before and after ESA treatment with and without swelling treatment . However since there was no influence of the swelling treatment, we report on samples which are generally subjected to the swelling treatment (XPS spectra was omitted).

Since the same XPS spectra were obtained regardless of the treatment time in ESA treatment, XPS spectr a both untreated before ESA treatment and treated for 120 minutes were shown in Fig.1. By ESA treatment, a large change occurs in the peaks around $\mathrm{O} 1 \mathrm{~s}(533 \mathrm{eV})$ and $\mathrm{C} 1 \mathrm{~s}$ $(285 \mathrm{eV})$.

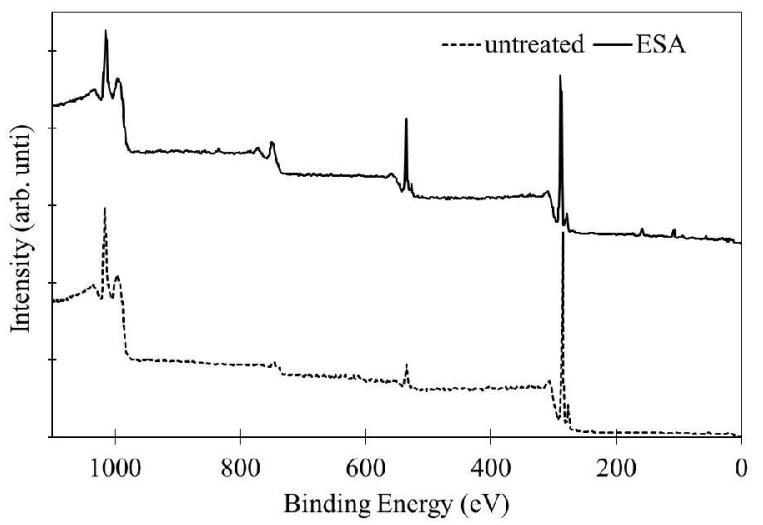

Fig.1 XPS spectra of untreated PP resin and PP resin surface treated for 120 minute $\mathrm{s}$ by ESA

First, $\mathrm{C} 1 \mathrm{~s}$ spectrum was investigated carefully. It is possible to know the presence of carboxyl group $(-\mathrm{COOH})$, carbonyl group $(-\mathrm{C}=\mathrm{O})$, and so on $[7,8]$. An example of the analysis is shown in Fig.2. In the presence of $-\mathrm{C}=\mathrm{O}$ or $-\mathrm{C}-\mathrm{O}$, the base of the $\mathrm{C} 1 \mathrm{~s}$ spectrum spreads in the direction of high binding energy.

Here, C1s spectrum before treatment and $\mathrm{C} 1 \mathrm{~s}$ spectrum treated with ESA for 120 minute s are shown in Fig.3. Additionally separated spectra on before treatment are shown in Fig.4, and those on after treatment are shown in Fig.5. Comparing both spectra of Fig .4 and Fig. 5, -C-O bond increases slightly and $-\mathrm{C}=\mathrm{O}$ bond occurs slightly, too. As they are almost the same as the peak position and charge -up amount, $-\mathrm{COOH}$ and $-\mathrm{C}=\mathrm{O}$ contain little. From the result, it can be said that carbon $(\mathrm{C})$ is not combined with oxygen $(\mathrm{O})$, that is, it is not oxidized. In addition, the $\mathrm{C} 1 \mathrm{~s}$ peak height is decreased by ESA treatment, that is, the carbon (C) concentration is lowered. It is thought that organic matter adhering to the surface was decomposed and removed by ESA treatment.

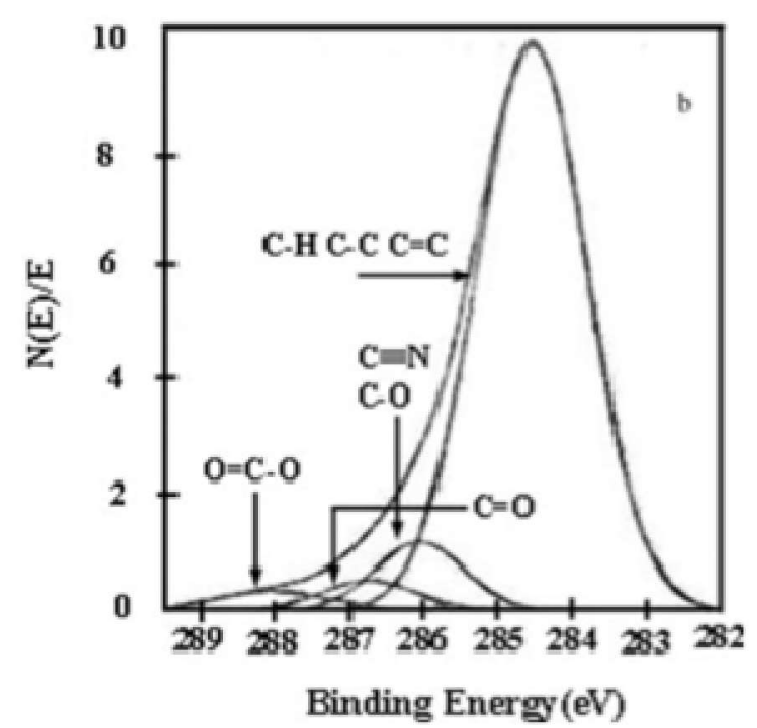

Fig.2 Example of C1s XPS spectra after oxidizing by Zenglin Wang et al.[7]

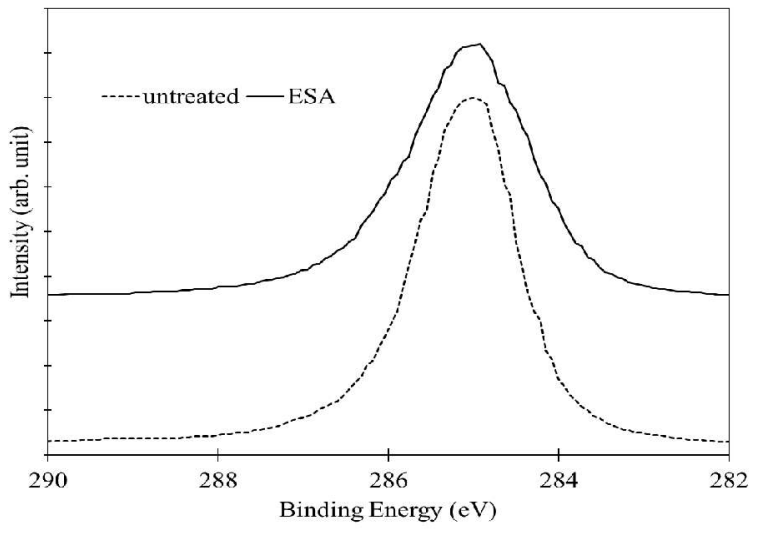

Fig.3 C1s XPS spectra of untreated PP resin and PP resin surface treated for 120 minute by ESA

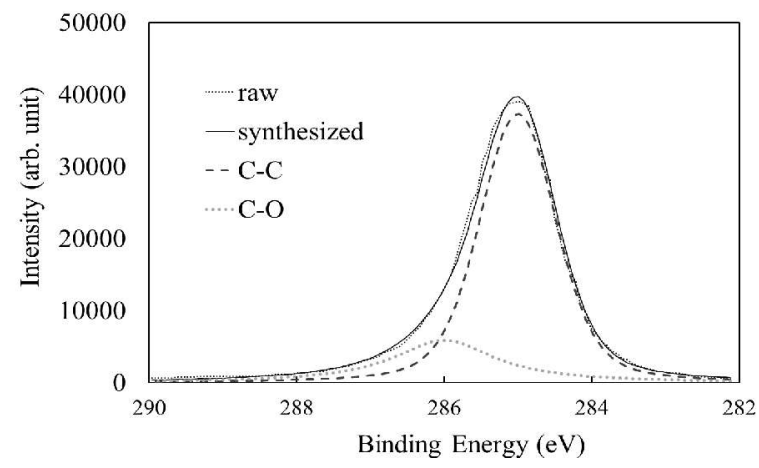

Fig.4 Separated C1s XPS spectra of untreated PP resin 


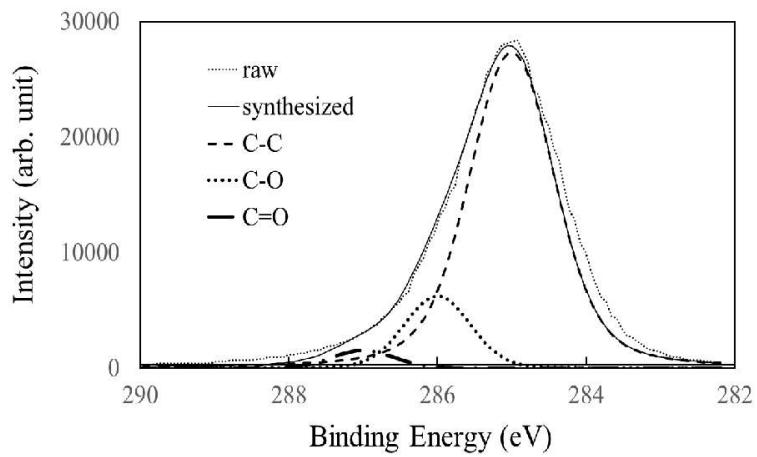

Fig.5 Separated C1s XPS spectra of PP resin surfaces treated for 120 minutes by ESA

Next O1s spectrum was investigated, too. Fig. 6 shows O1s spectr a before and after ESA treatment for 120 minutes. Additionally separated spectra on before treatment are shown in Fig. 7, and those on after treatment are shown in Fig.8.

Although oxygen is not originally contained in PP resin, when it is left in the atmosphere for a short period of time , it will inevitably adsorb water and organic matter as impurities and oxygen will be present along with it. Therefore, slight oxygen is detected on the surface before treatment. Comparing both spectra of Fig.7 and Fig. 8, it can be said that oxygen atoms $(\mathrm{O})$ increased due to the fact that the peak was about 6 times higher by ESA treatment. In addition, most of the $\mathrm{O}$ peak of ESA treatment is a hydroxyl group ( $-\mathrm{OH})$.

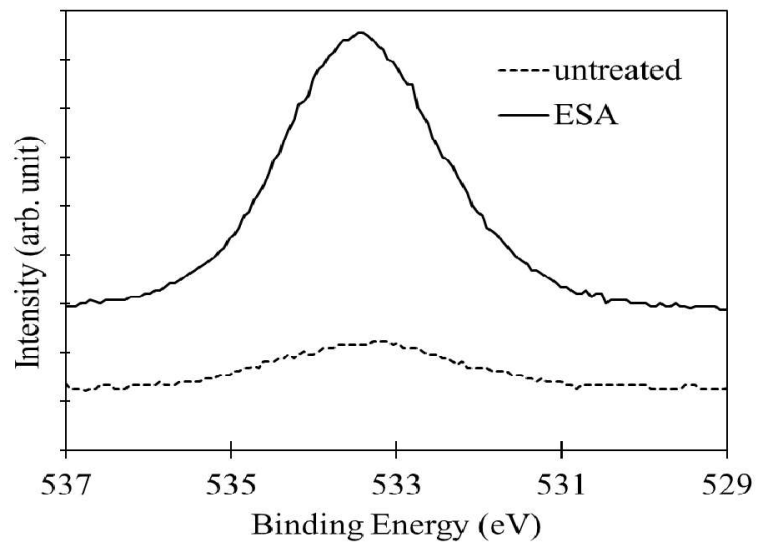

Fig.6 O1s XPS spectra of untreated PP resin and PP resin surface treated for 120 minute $\mathrm{s}$ by ESA

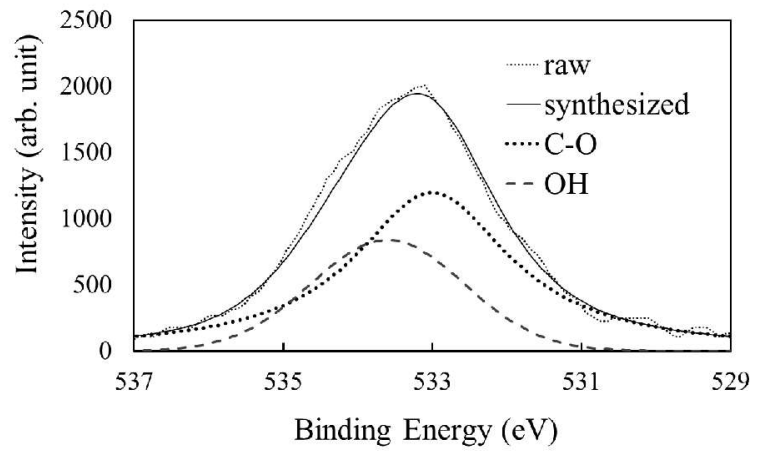

Fig.7 Separated O1s XPS spectra of untreated PP resin

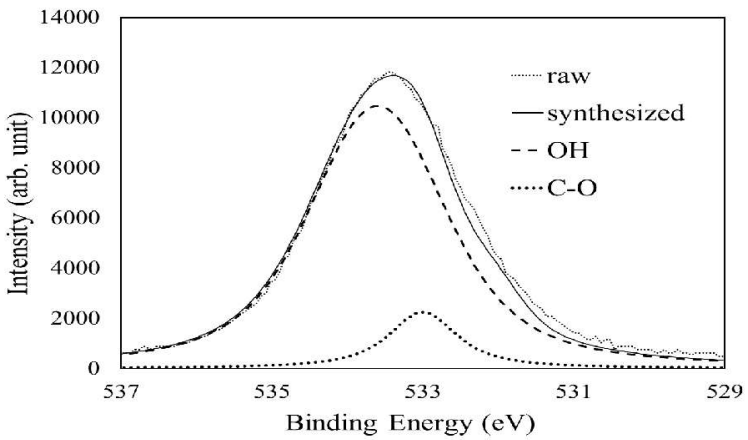

Fig.8 Separated O1s XPS spectra of PP resin surfaces treated for 120 minutes by ESA

\section{(2) ABS resin}

Since the same XPS spectra were obtained regardless of treatment time in ESA treatment, XPS spectra both untreated before ESA treatment and treated for 30 minut es were shown in Fig.9. As the result of ESA treatment, large changes are generated in the peaks around O1s (533 $\mathrm{eV})$ and $\mathrm{C} 1 \mathrm{~s}(285 \mathrm{eV})$ as $\mathrm{PP}$ resin.

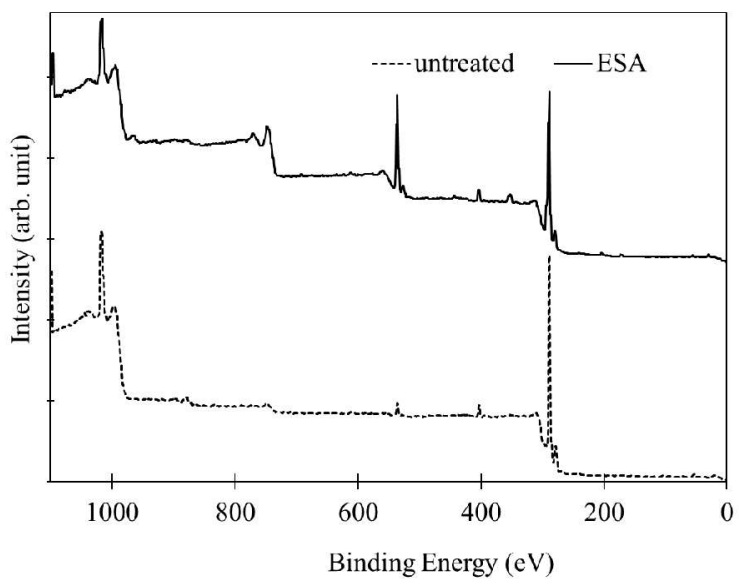

Fig.9 XPS spectra of untreated ABS resin and ABS resin surface treated for 30 minute $\mathrm{s}$ by ESA

On investigating the $\mathrm{C} 1 \mathrm{~s}$ spectra, it is almost the same as the peak position, the width at half maximum, and amount of charge -up as PP resin, and there exist no other peaks such as carboxyl group $\quad(-\mathrm{COOH})$ and carbonyl group $(-\mathrm{C}=\mathrm{O})$ as shown in Fig.2 . Thus carbon $(\mathrm{C})$ is not oxidized. And $\mathrm{C} 1 \mathrm{~s}$ peak height is decreased by ESA treatment. It is thought that organic matter adhering to the surface was also decomposed and removed by ESA treatment. From the O1s spectra, the oxygen $(\mathrm{O})$ is increased by ESA treatment as PP resin.

\subsection{FT-IR analysis}

Fig.10 and Fig.11 show the results of FT -IR analysis of $\mathrm{PP}$ resin and ABS resin surface under the same processing conditions as those used for XPS analysis. 


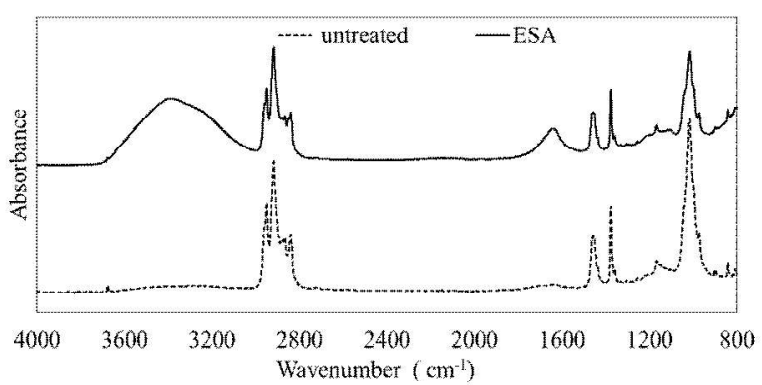

Fig.10 FT-IR spectra of untreated PP resin and PP resin surface treated for 120 minute $\mathrm{s}$ by ESA

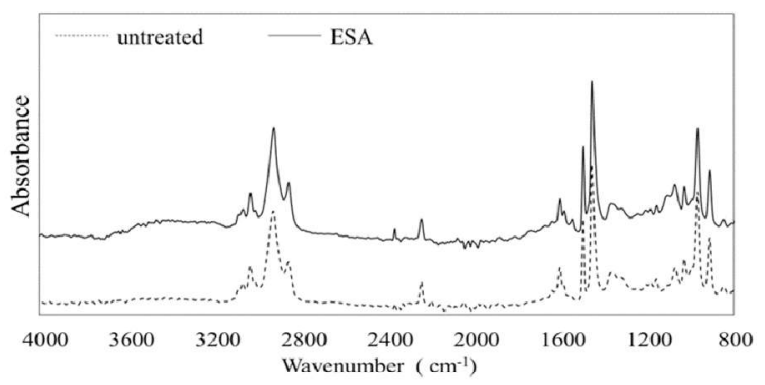

Fig.11 FT-IR spectra of untreated ABS resin and ABS resin surface treated for 30 minute $\mathrm{s}$ by ESA

It was confirmed that $-\mathrm{OH}$ group in the range of 3100 to $3600 \mathrm{~cm}^{-1}$ was greatly increased in FT -IR spectra about both of $\mathrm{PP}$ and $\mathrm{ABS}$ resin . In particular, the $\mathrm{OH}$ group on the PP resin showed a remarkable change. This is consistent with the result of XPS analysis. On PP resin, $\mathrm{C}=\mathrm{O}$ peak near $1650 \mathrm{~cm}^{-1}$ is increased by ESA treatment. It can be said that $-\mathrm{COOH}$ is slightly created as the distortion in C1s spectrum in XPS analysis.

3.3 Oxidation reaction on the surface of PP resin and ABS resin

(1) PP resin

The structure of PP resin is showing Fig.12 and has a tertiary carbon in the main chain. Since tertiary carbon has lower binding energy than primary and secondary carbon, it is susceptible to be oxidized, and the main chain is cut to decrease the molecular weight $[9,10]$.<smiles>CCCC(C)CC(C)C</smiles>

Fig.12 PP resin structure

As the sulfuric acid concentration was as high as 92 $\mathrm{wt} \%$, the presence and quantitative determination of 2-propanol could not be done. However, as understood from XPS and FT -IR analyses, hydroxyl group ( $-\mathrm{OH})$ occurs on the surface of PP resin, and PP resin is dissolved in ESA solution and roughened at the actual ESA treatment [ 5]. Therefore we suppose that the tertiary carbon is cleaved and is oxidized as follows Fig. 13.

Fig. 13 shows a model in which 2 -propanol is liberated, but the concentration of sulfuric acid is as high as $92 \mathrm{wt} \%$, and the presence and quantitative determination of 2-propanol cannot be done. Alcohol having a carbon number larger than that of 2 -propanol may be liberated.<smiles>CCC(C)C(C)CO</smiles>

(on PP surface)<smiles>CC(C)O</smiles>

(in the solution)
Fig.13 PP resin structure

(2)ABS resin

ABS resin is a terpolymer of acrylonitrile, butadiene, and styrene, and the structure is generally written as showing in Fig.1 4. However, polybutadiene particles disperse in acrylonitrile-styrene copolymer as the matrix phase actually. Therefore, when it is etched with chromic acid, the butadiene part $\mathrm{s}$ are preferentially dissolved [11].

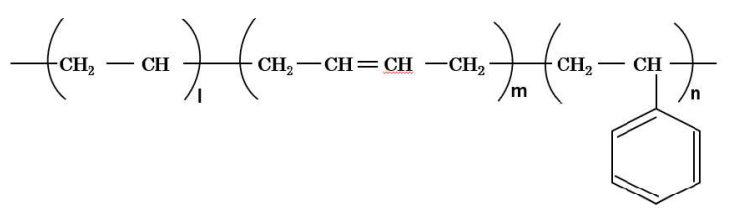

Fig.14 ABS resin structure

Since acrylonitrile -styrene resin contains tertiary carbons as PP resin, it is considered that hydroxyl group $(-\mathrm{OH})$ occurs at the carbon site.

\section{CONCLUSION}

It is possible for ESA treatment to enable PP resin and ABS resin to be plated with adhesion which can withstand practical use, although PP resin cannot be plated up to now and ABS resin is conventionally pretreated with chromic acid. That is because the surface of the resin can be changed hydrophilic by ESA treatment. We investigated the hydrophilization behavior from the viewpoint of functional group and found that the appearance of hydroxyl group ( $\quad-\mathrm{OH})$ must be created hydrophilic mainly.

In order to create strong adhesion between the resin surface and the plating film, it is necessary to have irregularities on the surface of the resin and to make the hydrophilization as discussed in this paper. We intend to discuss the importance of linking these two phenomena occurred simultaneously in ESA treatment and the application to other resins in the future.

\section{ACKNOWLEDGEMENT}

The authors would like to thank Mr. Takuya Maruko, Sakamoto Laboratory, Chiba Institute of Technology for cooperation in XPS analysis, and Mr. Yuzuki Yamamoto, Kurita Water Industries Ltd., for cooperation on the experiments related to this research. 


\section{REFERENCES}

[1] T. Hasegawa, H. Narita, the $11^{\text {th }}$ conference on manufacturing and machine tool division, Japan Soc. of Mech, Eng., D35 (2016).

[2] T. Nagai, H. Yamakawa, M. Uchida, T. Ohtsu, N. Ikemiya, IEICE Technical Report, 108(87), 41-45 (2008).

[3] T. Nagai, H. Yamakawa, M. Uchida, T. Ohtsu, N.

Ikemiya, and H. Morita, Solid State Phenomena ， 187, 109-112 (2012).

[4] Y. Nakabayashi, Y. Umeda, T. Nagai, K. Tashiro, H. Honma and H. Kouzai, Trans. Mat. Res. Soc. Japan (publishing decision).

[5] Y. Umeda, Y . Nakabayashi, T . Nagai, K. Tashiro, H . Honma, and Y. Sakamoto, Material Sci. and Tech. Japan, 55(6), (2018) (publishing decision).

[6] A. Otagawa, Jitsumu Hyomen Gijutsu, 27(11), 561-566 (1980).

[7] Zenglin Wang, Zhixin Li, Yue He, and Zhixiang Wang, $E C S$, 158(11), 664-670 (2011)

[8] Y. Nakayama, T. Takahagi, and F. Soeda, J. Polym. Sci., Part A, 26, 559-572 (1988)

[9] H. Yamanoi, Materiaru Raifu Gakkaishi, $\quad$ 19(3), 103-108 (2007)

[10] T. Iizuka, Doctoral thesis of Kyushu Univ. (2016)

[11] K. Kita, Journal of Surf. Finishing Soc. Japan, 64(12), 622-627 (2013)

(Received October 24, 2018; Accepted December 6, 2018; Published Online February 1, 2019) 